\title{
Nanowire arrays as cell force sensors to investigate adhesin-enhanced holdfast of single cell bacteria and biofilm stability
}

\author{
Prasana. K. Sahoo ${ }^{1}$, Richard Janissen ${ }^{1,2}$, Moniellen P. Monteiro1, Alessandro \\ Cavalli ${ }^{3}$, Duber M. Murillo ${ }^{1}$, Marcus V. Merfa ${ }^{4}$, Carlos L. Cesar ${ }^{5}$, Hernandes F. \\ Carvalho $^{6}$, Alessandra A. de Souza ${ }^{4}$, Erik P.A.M. Bakkers ${ }^{3}$, Monica A. Cotta ${ }^{\text {l, * }}$ \\ ${ }^{1}$ Applied Physics Department, Institute of Physics 'Gleb Wataghin', State University of Campinas, 13083-859, Campinas, São Paulo, \\ Brazil \\ ${ }^{2}$ Kavli Institute of Nanoscience, Delft University of Technology, 2628 CJ Delft, The Netherlands \\ ${ }^{3}$ Applied Physics Department, Eindhoven University of Technology, 5600 MB Eindhoven, The Netherlands \\ ${ }^{4}$ Citrus Center APTA 'Sylvio Moreira', Agronomic Institute of Campinas, 13490-970, Cordeirópolis, São Paulo, Brazil \\ ${ }^{5}$ Quantum Electronics Department, Institute of Physics 'Gleb Wataghin', State University of Campinas, 13083-859, Campinas, São \\ Paulo, Brazil \\ ${ }^{6}$ Structural and Functional Biology Department, Institute of Biology, State University of Campinas, 13083-865, Campinas, São Paulo, \\ Brazil
}

\section{Supplemental Information}

\section{Material and Methods}

\section{Supporting figures and tables}

Figure S1 $X$. fastidiosa cell size distribution

Figure S2 FESEM images (top view) of $X$. fastidiosa on flat InP surface and InP NW arrays

Figure S3 FESEM images of single $X$. fastidiosa cells adhered to InP nanowires

Figure S4 Ex-vivo CLSM-based force measurements of singly adhered $X$. fastidiosa cells

Figure S5 Ex-vivo forces of adhered $X$. fastidiosa single cells as a function of time

Figure S6 Ex-vivo force measurements for single cell with cell impact on nanowire

Figure S7 FESEM images of biofilm formed on nanowire arrays

Figure S8 $X$. fastidiosa cell adhesion on bare and XadA1-coated InP surfaces and NW arrays

Figure S9 Force calibration of InP nanowires as a function of NW length and diameter

Table S1 Adhesion force values obtained for all experimental conditions

Table S2 Adhesion forces of single cell biofilm anchors

\section{Supporting movies (online)}

Movie 1 Movement of single $X$. fastidiosa cells

Movie 2 Horizontally static single $X$. fastidiosa cells on NW arrays

Movie 3 Horizontal movement of single $X$. fastidiosa cells on nanowire (NW) arrays

Movie 4 Vertical movement of single $X$. fastidiosa cells on NW arrays

Movie 5 Vertically static single $X$. fastidiosa cells on NW arrays

Movie $6 X$. fastidiosa biofilm 3D - rotating view

Movie 7 Horizontally static single $X$. fastidiosa cells on +XadA1 NW arrays

Movie 8 Horizontal movement of single $X$. fastidiosa cells on + XadA1 NW arrays

Movie 9 Vertical movement of single $X$. fastidiosa cells on +XadA1 NW arrays

Movie 10 Vertically static single $X$. fastidiosa cells on + XadA1 NW arrays 


\section{Material and Methods}

Chemicals and reagents. All buffers and chemicals used in this study were purchased from SigmaAldrich, USA, unless otherwise mentioned therein.

\section{Indium Phosphide nanowire array growth.}

Nanowires were grown in a low-pressure Aixtron Closed Coupled Showerhead (CCS) MOVPE machine, with Hydrogen $\left(\mathrm{H}_{2}\right)$ used as carrier gas for precursors. The total flow was $61 / \mathrm{min}$ at a pressure of 50 mbar. Nanowires were grown on (111) InP substrates with the vapor-liquid-solid (VLS) method, in which Au droplets act as catalysts. $8 \mathrm{~nm}$ thick, $180 \mathrm{~nm}$ wide Au disks were patterned, with a square symmetry and a pitch of $513 \mathrm{~nm}$, by nanoimprint lithography on a 2 inch wafer. ${ }^{1}$ The $90 \mathrm{~nm}$ nanowire diameter was defined by the amount of Au catalyst. Before nanowire growth, an annealing step at 510 ${ }^{\circ} \mathrm{C}$ under $\mathrm{PH}_{3} / \mathrm{H}_{2}$ atmosphere was performed to remove organic residues from the nanoimprint process. Tri-methyl-indium (TMI) and phosphine $\left(\mathrm{PH}_{3}\right)$ were used as group III and V precursors. p-doped nanowires were obtained by doping in-situ by diethyl-zinc (DEZn) with a molar fraction of $6 \times 10^{-6}$. $\mathrm{HCl}$ was used in-situ to suppress tapering of the nanowires, with molar fraction constant during growth. $^{2}$ Growth times of p-doped nanowire were $18 \mathrm{~min}$, after a short $1 \mathrm{~min}$ undoped stem. ${ }^{3}$ Nanowires grew vertically, perpendicular to the substrate, in the $<111>$ direction. The typical diameter and length of the nanowires in the array were 90 and $1500 \mathrm{~nm}$, respectively. For bacterial adhesion studies, the substrates were cut into rectangular pieces of approximately $2 \times 3 \mathrm{~mm}$.

Substrate materials and cleaning process. For all conducted bacterial adhesion experiments, planar, undoped, (100)-oriented InP wafer substrates (AXT, USA), grown InP nanowire arrays and 22 x 22 $\mathrm{mm}$ borosilicate cover glasses (\#0, Menzel GmbH, Germany) were cleaned to remove inorganic as well as organic contamination, and sterilized in a final step. To do so, the substrates were cleaned with acetone, isopropanol and deionized water, and dried with a gentle nitrogen flow. Prior to the bacterial adhesion studies, the substrates were sterilized by oxygen plasma (SE80, Barrel Asher Plasma Technology, USA) for $15 \mathrm{~min}\left(50 \mathrm{sccm} \mathrm{O}_{2}, 100 \mathrm{~W}, 100 \mathrm{mTorr}\right)$.

Cloning, expression and purification of Xf.XadA1 membrane protein. The procedure used here was previously described in Moreau et al. ${ }^{4}$ The XF.XadA1 sequence ORF Xf1257 (3015bp), which encodes the Xylella fastidiosa surface adhesion protein Xf.XadA1 (1005 aa), was amplified from genomic DNA by PCR using specific primers. The "head" domain of Xf.XadA1, beginning at position 
50 aa and ending at position 225 aa, was constructed using the primers XF.XadA $1_{\text {forward }}\left(5^{6}-\right.$ CATAGCTAGCGGTCTTGCGCTTACAA-3') and XF.XadA1 $1_{\text {reverse }}$ (5'-TGGAATTCGGCAATCG TCTTCACC-3') containing the NheI and EcoRI restriction enzyme sites, respectively. The PCR amplification product was cloned into the expression vector pET28a(+) (Novagen, USA); an additional $\mathrm{N}$-terminal six-histidines tag and a thrombin protease site to the coding sequence were included in this vector using the NheI and EcoRI restriction sites. The cloned domains were overexpressed in E. coli C43 (DE3) (Avidis) strain. Cells were grown at $37^{\circ} \mathrm{C}$ in 1 liter of LB media, supplemented with $0.2 \%$ glucose and containing kanamycin $(30 \mu \mathrm{g} / \mathrm{mL})$ until optical absorbance of $\mathrm{DO}_{600}$ of $0.6-0.8$ was reached. Recombinant proteins were induced by the addition of $1 \mathrm{mM}$ IPTG (isopropyl- $\beta$-d-1thiogalactopyranoside) followed by cultivation for $4 \mathrm{~h}$ at $25^{\circ} \mathrm{C}$ and $200 \mathrm{rpm}$. The culture was harvested by centrifugation $\left(3000 \mathrm{~g}, 15 \mathrm{~min}, 4^{\circ} \mathrm{C}\right)$; sedimented cells were resuspended in $50 \mathrm{~mL}$ of buffer A (50 $\mathrm{mm}$ Tris- $\mathrm{HCl}, \quad 150 \mathrm{~mm} \mathrm{NaCl}, \quad \mathrm{pH} \quad 8.0)$ plus $1 \mathrm{mg} / \mathrm{mL}$ lysozyme and $1 \mathrm{mM}$ phenylmethanelsulfonylfluoride (Sigma, St Louis, USA) and incubated for $30 \mathrm{~min}$ on ice. The lysates were disrupted by sonication and the unbroken cells and debris were removed by centrifugation (27000 $g, 40 \mathrm{~min}, 4^{\circ} \mathrm{C}$ ). The Xf.XadA1 protein purification was performed by affinity chromatography using a Ni-NTA column (Qiagen, Hilden, Germany), equilibrated with buffer A. The purified XadA1 proteins were eluted with five column volumes of buffer A containing $250 \mathrm{mM}$ imidazole and the degree of purity was estimated by SDS-PAGE. Subsequently, the purification step the N-terminus His $_{6}$-tag of recombinant proteins were removed using a thrombin cleavage kit (Novagen, USA).

InP surface functionalization with XadA1 adhesin. The applied surface functionalization procedure is based on a previously described methodology ${ }^{4}$ with minor modifications. Here, the cleaned and oxygen plasma treated InP substrates were incubated for $12 \mathrm{~h}$ in anhydrous DMSO (dimethyl sulfoxide) containing $5 \mathrm{M}$ ethanolamine hydrochloride for surface amination. The substrates were then washed three times with deionized water and dried using a gentle nitrogen flow. These freshly prepared aminated substrates were further covalently PEGylated by incubation in amino-reactive heterobifunctional NHS-PEG-COOH (MW 3.400, LaysanBio, USA) in anhydrous chloroform containing $0.5 \%(\mathrm{v} / \mathrm{v})$ triethylamine for $1 \mathrm{~h}$ at room temperature. After the PEGylation process, the supports were washed five times with deionized water and dried with a gentle nitrogen flow. For covalent XadA1 protein attachment, $1 \mu \mathrm{M}$ of the protein was added to the PEGylated InP substrates and bound via peptide binding in $100 \mathrm{mM}$ MES (2-(N-morpholino)ethanesulfonic acid, $\mathrm{pH}$ 4.7) buffer containing $50 \mathrm{mM}$ EDC (1-Ethyl-3-(3-dimethylaminopropyl)-carbodiimide) for $1 \mathrm{~h}$ reaction time at room temperature in humid atmosphere. After XadA1 immobilization, the substrates were washed five times with PBS buffer ( $\mathrm{pH}$ 7.4) and immediately used for the bacterial adhesion studies. The $1 \mathrm{~h}$ 
reaction time and several washing steps ensure the complete inactivation of EDC-activated carboxylic groups before ex-vivo bacterial experiments. ${ }^{5,6}$

Bacteria strains. Xylella fastidiosa wild type strain 9a5c and Green Fluorescent Protein (GFP) expressing strain 11399 were used in this study. Periwinkle Wilt broth (PW) ${ }^{7}$ with Bovine Serum Albumin (BSA) was used as bacterial growth media.

Bacteria extraction and inoculum preparation. The extraction and growth of $X$. fastidiosa strains from Citrus variegated chlorosis (CVC) symptomatic sweet orange trees were conducted as previously described. ${ }^{8,9}$ Harvested cells were resuspended in PBS (pH 7.4) buffer and the concentration was adjusted to $\mathrm{OD}_{600}=0.3$. Afterwards, the strains were grown in $\mathrm{PW}$ broth and incubated at $28^{\circ} \mathrm{C}$ for seven days in a rotary shaker at $180 \mathrm{rpm}$.

Bacterial growth. Bacterial inoculum in PW broth media with a concentration of $1 \times 10^{7} \mathrm{CFU} \mathrm{mL}^{-1}$ were used for all adhesion experiments. InP nanowire arrays $\left(2 \times 3 \mathrm{~mm}^{2}\right)$ were placed inside a custom made Teflon chamber (with $10 \mathrm{~mm}$ diameter and $5 \mathrm{~mm}$ in height) and $400 \mu 1$ of inoculum were added. After covering the chamber with a sterilized borosilicate cover glass, the assembly was incubated inside a bacterial oven (410/3NDR, Nova Ética, Brazil) at $28^{\circ} \mathrm{C}$. After chosen growth times $(24 \mathrm{~h}$ and $168 \mathrm{~h}$ ), the whole system was taken for ex-vivo CLSM measurements. For scanning electron microscope studies, PW broth media was gently removed and the samples were washed three times with deionized water to completely remove the constituents of the culture media and non-attached biofilms; the samples were dried gently in nitrogen flow and temporarily stored at $4-8^{\circ} \mathrm{C}$ before measurements.

Polyclonal XadA1 antibody production. The $X$. fastidiosa polyclonal IgG antibody (Rheabiotech, Brazil) against XadA1 (further called anti-XadA1) was obtained by immunization of New Zealand White Rabbits based on the protocol of Caserta et al. ${ }^{9}$ Briefly, $150 \mu \mathrm{g}$ purified XadA1 proteins were mixed with Freund's complete adjuvant and injected into individual rabbits. The proteins solution was injected two more times, at 10 and 20 days after the first injection. The quality of the antibodies was verified by performing a direct ELISA (enzyme-linked immunosorbent assay), using the target XadA1 proteins as antigens and PBS as the negative control.

Immunolabeling of XadA1 adhesin. The immunofluorescence-based identification and localization of XadA1 adhesines was performed using polyclonal anti-XadA1 antibodies in combination with 
Alexa488 conjugated secondary goat anti-rabbit IgG antibodies (\#A11008, Invitrogen, USA). To do so, unfixed $9 \mathrm{a} 5 \mathrm{c}$ bacteria strain samples with $24 \mathrm{~h}$ of growth at $28^{\circ} \mathrm{C}$ were washed twice for 5 min with phosphate-buffered saline (PBS, $\mathrm{pH}$ 7.4) to remove the constituents of the culture media and planktonic as well as non-attached clusters. In order to reduce unspecific adsorption of the antibodies, the samples were passivated with PBS/BSA buffer (PBS, containing 3\% (w/v) BSA) for $1 \mathrm{~h}$ at room temperature. After washing the samples three times with PBS-T buffer (PBS, containing $0.01 \%(\mathrm{v} / \mathrm{v})$ Tween20) for $2 \mathrm{~min}$ each, $10 \mu \mathrm{g} \cdot \mathrm{mL}^{-1}$ of anti-XadA1 antibodies in PBS/BSA buffer were added to the samples and incubated for $1 \mathrm{~h}$ at $37^{\circ} \mathrm{C}$. The samples were then washed twice for $10 \mathrm{~min}$ with PBS-T buffer and once with PBS buffer for $5 \mathrm{~min}$. For fluorescence labeling of the anti-XadA1 antibodies, 1 $\mu \mathrm{g} \cdot \mathrm{mL}^{-1}$ of Alexa488-labeled secondary antibody was added to the samples in PBS/BSA buffer and again incubated for $1 \mathrm{~h}$ at $37^{\circ} \mathrm{C}$, protected from light. In a final step, the samples were washed twice for 2 min with PBS-T buffer, twice with PBS buffer for 3 min and briefly with deionized water and then finally dried gently under nitrogen flow.

Lipid labeling process. For fluorescence-based labeling of lipid membranes, the commercial available PKH26 fluorescent linker kit was used (\#P9691, Sigma). Lipid staining was carried out after XadA1 immunolabeling. To do so, $4 \mu \mathrm{M}$ dye solution was added to the $9 \mathrm{a} 5 \mathrm{c}$ bacterial strain samples and incubated for $5 \mathrm{~min}$ at room temperature. Afterwards, the samples were washed twice with deionized water and gently dried under nitrogen flow.

Wide-field epifluorescence microscopy. For the study of cell density adhered to flat InP substrate, the GFP-expressing bacteria strain 11399 samples were measured using an epifluorescence microscope (Nikon TE2000U, USA) with a peltier-cooled back-illuminated EMCCD camera (IXON ${ }^{3}, 1024 \times 1024$ pixels, Andor, Ireland) with 100× oil-immersion objective (CFI APO TIRF, NA. 1.45, Nikon, USA). A $150 \mathrm{~W}$ Mercury-lamp with appropriate filter sets (AHF, Tübingen, Germany) for GFP excitation and emissions were used to record the fluorescence images of bacteria. The reported number of bacteria identified on the InP substrate is based on the analysis of $n=30$ measurements for 3 repetitions each.

Confocal laser scanning microscopy. Confocal Laser Scanning Microscopy (CLSM) measurements of bacteria adhered to nanowires and for immunolabeling studies on flat InP surface were carried out using a Zeiss LSM780-NLO confocal microscope (Carl Zeiss AG, Germany) with a 63× oil-immersion objective (Plan-Apochromat, NA. 1.4). The nanowire position and bacteria cells were simultaneously recorded from the $488 \mathrm{~nm}$ laser excitation in two different channels. The position of the individual nanowires of the arrays were captured from the reflection of laser line in one channel while the 
localization of bacteria was performed by measuring the fluorescence emission of GFP (505 - $540 \mathrm{~nm})$ in another channel. For analysis, the images were merged using Image J2 software.

The XadA1 distribution measurement via Alexa488-labeled secondary antibodies was examined using a laser line of $488 \mathrm{~nm}$. Similarly, $651 \mathrm{~nm}$ laser excitation was used for lipid distribution imaging of the same samples via the PKH26 dye. All imaging was performed with pinholes set to 1 airy unit for each channel with $512 \times 512$ px (nanowire with bacteria) and $1024 \times 1024$ px (flat surface) image resolution and distances of $370 \mathrm{~nm}$ for the z-stack. The 3D-image analysis of the nanowire with bacterial and biofilm architecture was performed with ImageJ2/Fiji in combination with Imaris software (Bitplane, USA). ${ }^{10}$ The reported observations were based on the analysis of $n>50$ image acquisitions.

Scanning electron microscopy of bacterial cells on InP surface. The cell-surface interaction and cell adhesion density of $X$. fastidiosa cells, the EPS deposition and filament formation on different InP substrates were investigated using high-resolution FESEM (Field Emission Scanning Electron Microscopy; FEI Inspect F50). In order to preserve the cell integrity, EPS components and filament shapes, the samples were analyzed using low electron beam energy $(1 \mathrm{keV})$ with short exposure times and secondary electron imaging mode. The use of Field Emission Gun (FEG) in the SEM system simultaneously provides high resolution and high contrast images with low electrostatic distortion, in general resulting in spatial resolution $<2 \mathrm{~nm}$. For cell adhesion density analysis, $n>100$ FESEM images were measured.

Image analysis. The measured time series of nanowire positions and cell positions were analyzed by ImageJ, ImageJ2/Fiji and Imaris software. ${ }^{10}$ For each time series of cellular force measurements, the positions of all nanowire tips in a video frame were tracked by considering them as a solid circular particles; the center of the circle was computed from the position of horizontal and vertical Feret diameters of a circular post, automatically by appropriately choosing the range of particle size in the particle analysis plugin of ImageJ2/Fiji (Fig S4). The automated particle tracking analyzer module simultaneously analyzes and records the position of numerous objects and combines spots as they move through time. This plugin was also used to measure bacteria that move in space by diffusion or motility, to find the location of post appearances and disappearances, and to calculate the lifetime of post during a time series. By comparing manual and automated results for selected images, we estimate that displacement deviations are smaller than $10 \%$. The results were used for analysis of bacteria position and movement as well as for nanowire force changes in magnitude and orientation, for a chosen set of nanowires in each video frame. The 3D images of nanowire and bacteria/biofilm position 
were developed thereby combining the vertical tracking position of two different channels for the 3D viewer plugin in ImageJ.

Force calculations. The magnitude and orientation of the deflection of nanowires, determined from their tip positions in the times series, was analyzed using the Image J software and translated into forces. According to the linear elasticity theory for hexagonal cross-section, ${ }^{12,13}$

$$
\mathrm{F}=\frac{3 \mathrm{EI}}{L^{3}} \Delta \mathrm{x}=\frac{15 \sqrt{3} E D^{4}}{256 L^{3}} \Delta \mathrm{x}
$$

where E depicts the Young's modulus of the nanowires, I corresponds to the second moment of inertia, $D$ specifies the diameter of the nanowire, $L$ identifies the length of the nanowire and $\Delta x$ determines the displacement of the tip of the nanowire. The parameters for corresponding InP nanowire arrays used in this study are: $\mathrm{D}=90 \mathrm{~nm}, \mathrm{~L}=1500 \mathrm{~nm}$, and Young's Modulus $\mathrm{E}=106.4 \mathrm{GPa}$ for InP (111). ${ }^{14} \mathrm{It}$ is important to emphasize that the nanowire diameter and length can be adjusted in order to probe a particular range of cellular forces (Fig S9).

Statistical analysis. For all employed statistical tests we used for the data selection Tukey's outlier filter of leveraging the Interquartile Range. ${ }^{15}$ This method is applicable to most ranges since it does not depend on distributional assumptions. It also ignores the mean and standard deviation, making it resistant to being influenced by the extreme values in the range. Bacterial density and length values as well as the described nanowire force distributions were plotted as Tukey box plots considering the described outlier filter. The statistical tests to analyze differences in bacterial density and length as a function of InP surface composition was performed using two-tailed t-test with a significance level of $p=0.0001$. Cellular adhesion forces exerting on nanowires dependent on InP surface composition, cell orientation and movement were analyzed using one-way two-tailed analysis of variance (ANOVA, $p=0.0001)$ with subsequent Tukey post-hoc test for statistical comparison. 


\section{Supporting figures and tables}

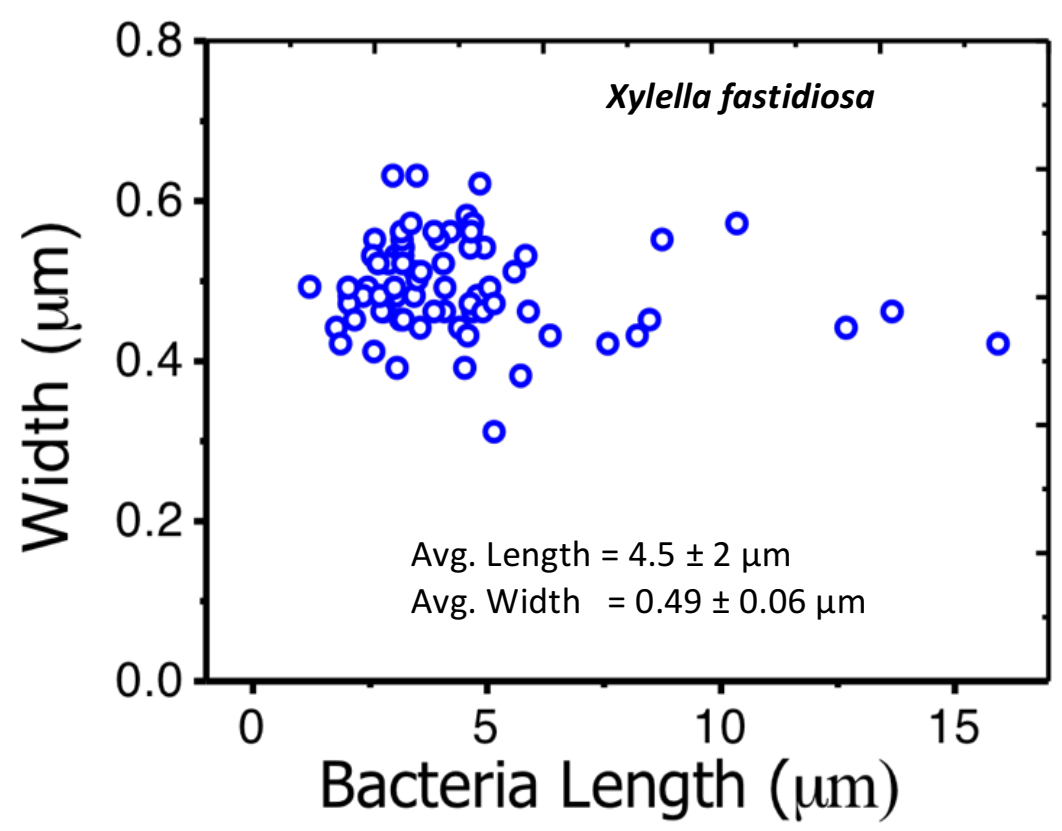

Figure S1. Size distribution of $X$. fastidiosa cells obtained from FESEM and WFM images. Average length and width are shown as inset. The longer cells are due to filamentation taking place in between clusters. ${ }^{8}$

A

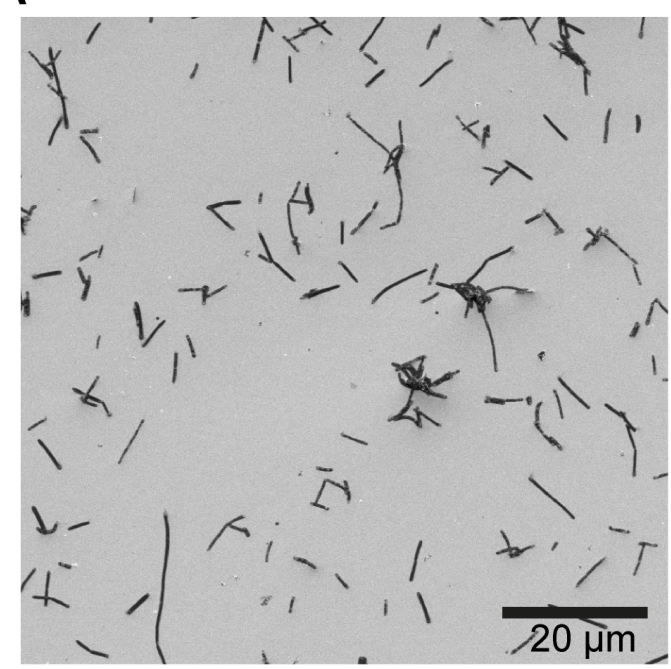

B

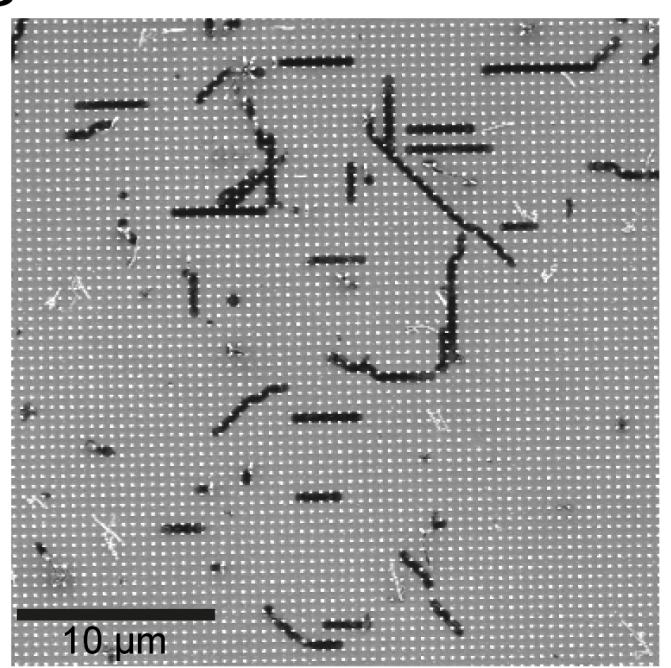

Figure S2. Large scale FESEM images of dry $X$. fastidiosa cells on (A) flat InP substrate and (B) InP nanowire arrays. The alignment of bacteria following the nanoscale topography is clearly observable; cells are attached within the vicinity of nanowires in the array. 


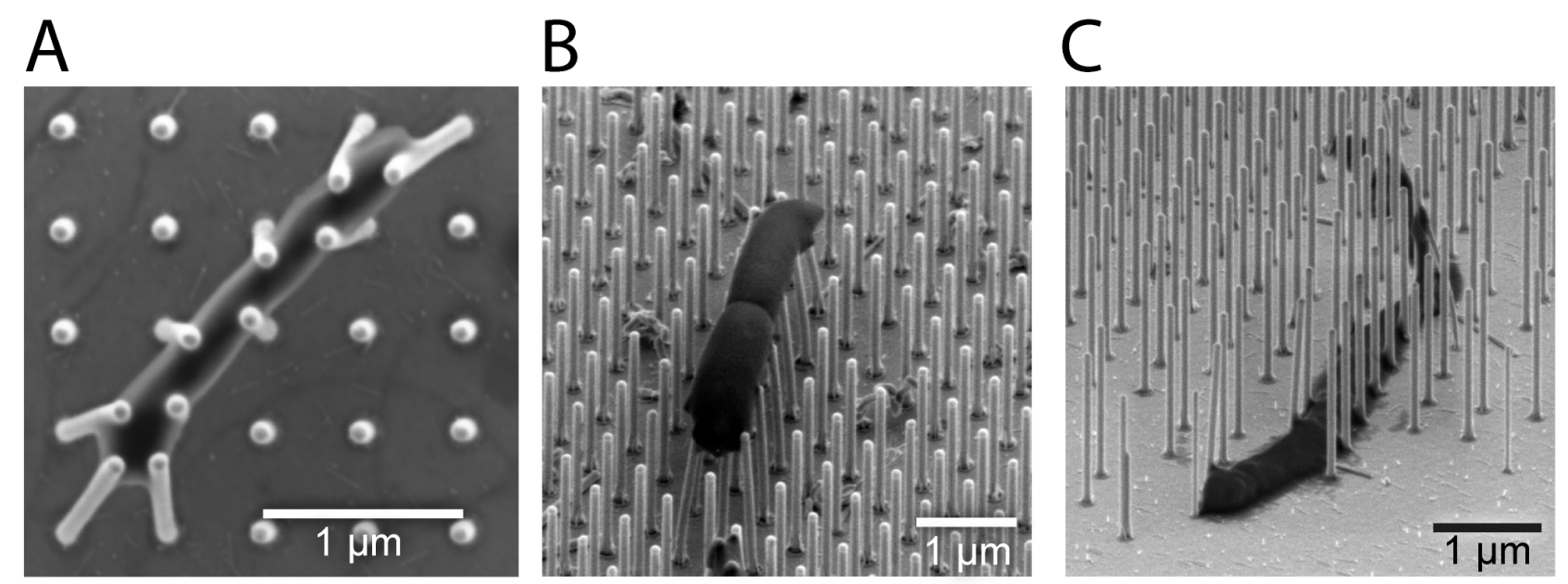

Figure S3. FESEM images of single $X$. fastidiosa cells on InP nanowire arrays. Perpendicular view (A) of a cell trapped by nanowire arrays, most likely via the secreted EPS matrix. Two daughter cells (B) attached on the top of the nanowire arrays. Cells on the bottom surface (D) within nanowire vicinity in the array. 
A

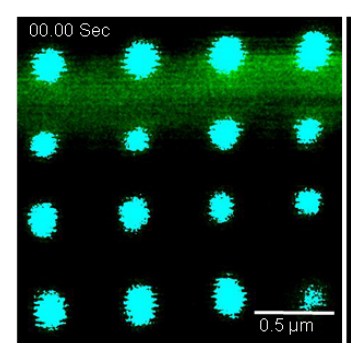

$\mathrm{B}$

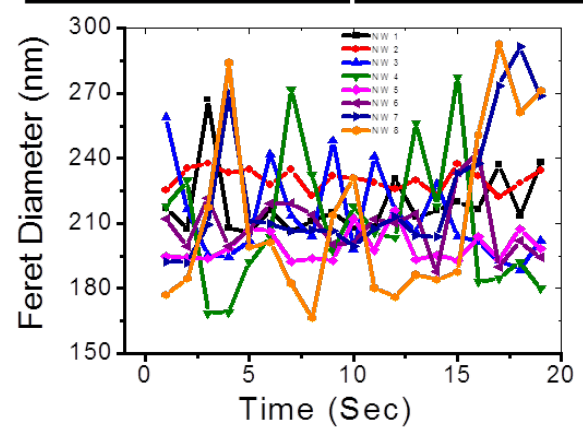

$\mathrm{D}$

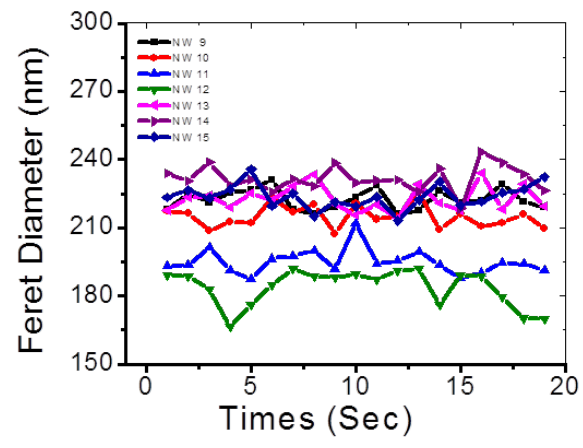

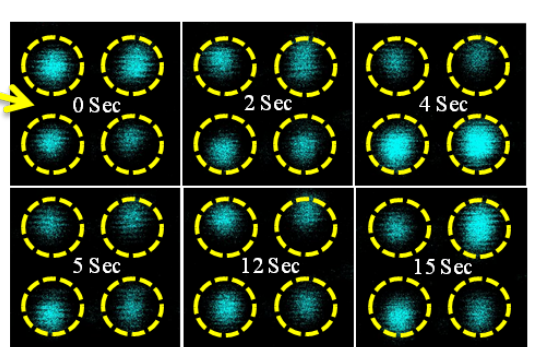

$\mathrm{C}$

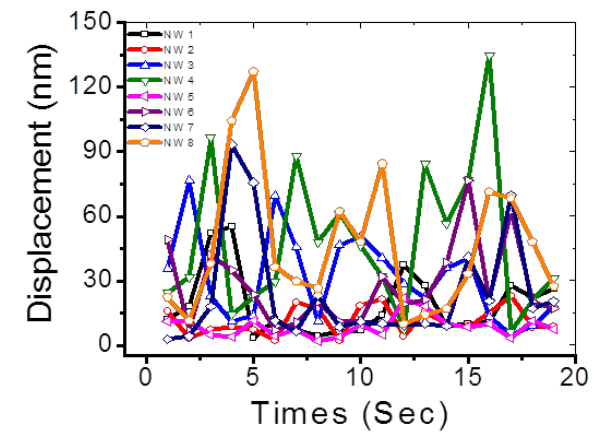

$\mathrm{E}$

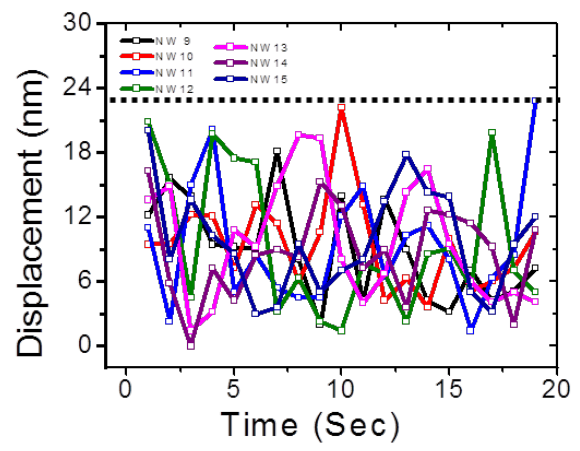

$\mathrm{F} * * * 0 \mathrm{Sec}$

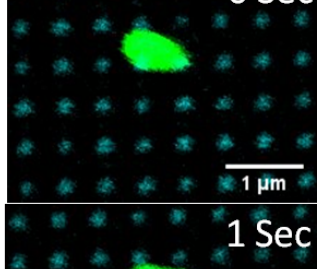

$1 \mathrm{Sec}$

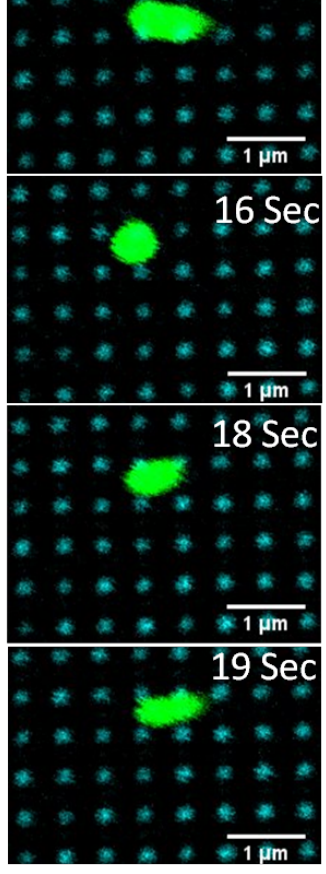

Figure S4. Ex-vivo force measurements for single cells. The CLSM images show X. fastidiosa cells adhered to InP nanowires (the images show the fluorescence of GFP and the reflected laser intensity at the nanowire tip). Moving bacterial cell attached horizontally (A) on nanowire arrays and selected region (yellow dotted Square; middle) shows how the nanowire top position changes upon bacterial movement (right side). Analysis of CLSM extracted video frames provided the time series of Feret diameters $(\mathbf{B}, \mathbf{D})$ and nanowire displacements $(\mathbf{C}, \mathbf{E}$; notice the different scales for the vertical axis) for $(\mathbf{B}, \mathbf{C})$ nanowires with bacteria attached and $(\mathbf{D}, \mathbf{E})$ standalone nanowires with no bacteria (used as control for the experiment). The dashed line in $(\mathbf{E})$ indicates the displacement associated with the noise limit force value $(5 \mathrm{nN})$. A vertically attached, moving cell and the temporal position recorded from the captured video $(\mathbf{F})$. 
A

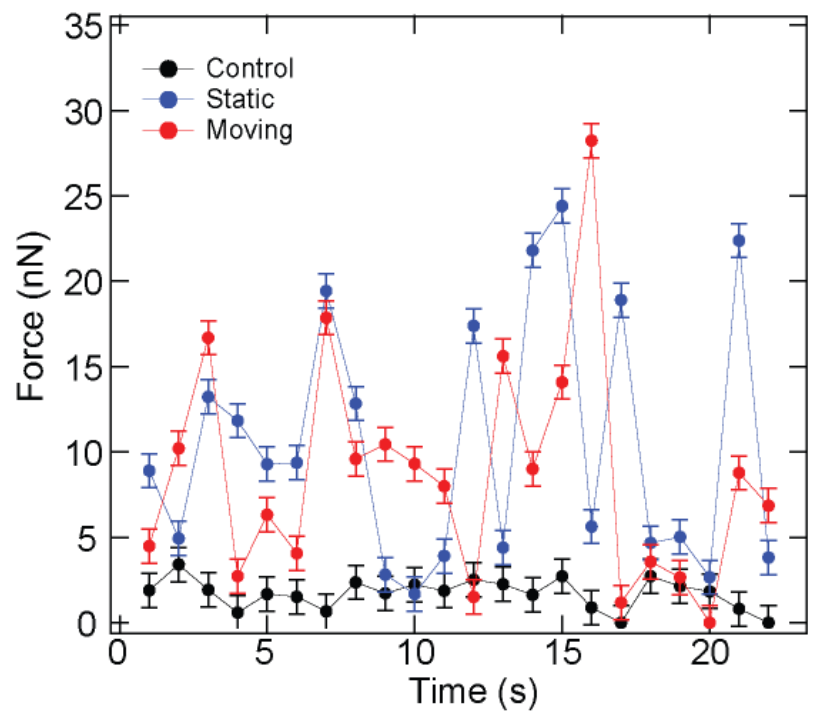

B

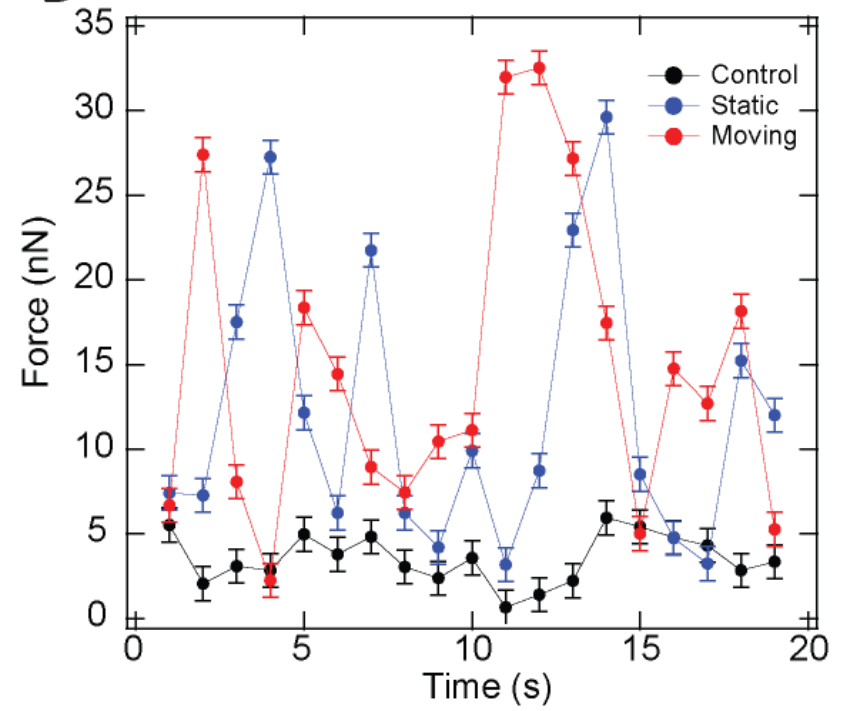

Figure S5. Ex-vivo force measurements for single cells. The graphs show the change in forces over time for control nanowires (black), static (blue) and moving (red) adhered bacterial cells in the horizontal (A) and vertical (B) configurations. The large fluctuations are mainly due to the 'stroboscope' effect caused by the slow laser scanning rate, when compared to the faster nanowire motion.

A

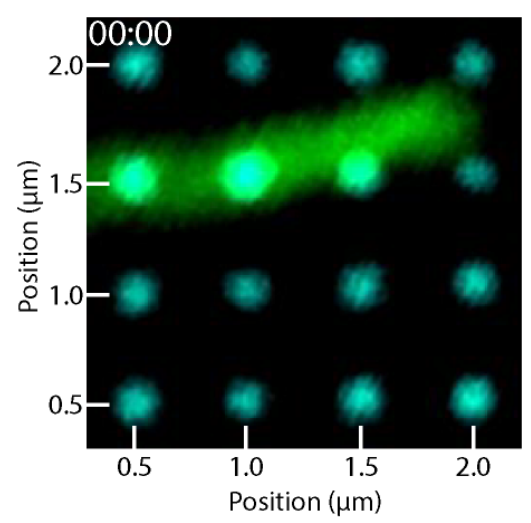

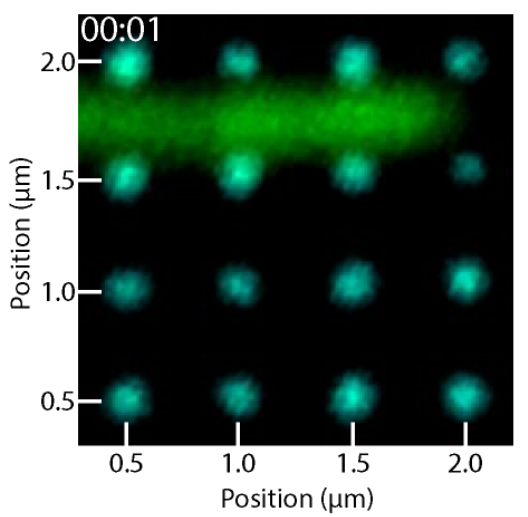

B

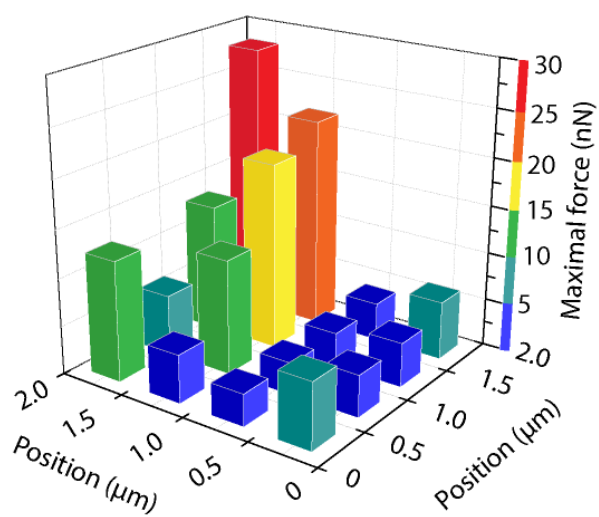

Figure S6. Ex-vivo force measurements for single cells. Recorded video frames (A) show the movement of bacterial cell with pole adhered to nanowire outside the image field of view. Corresponding maximum force values $(\mathbf{B})$ observed for each nanowire in the image (A). 
A

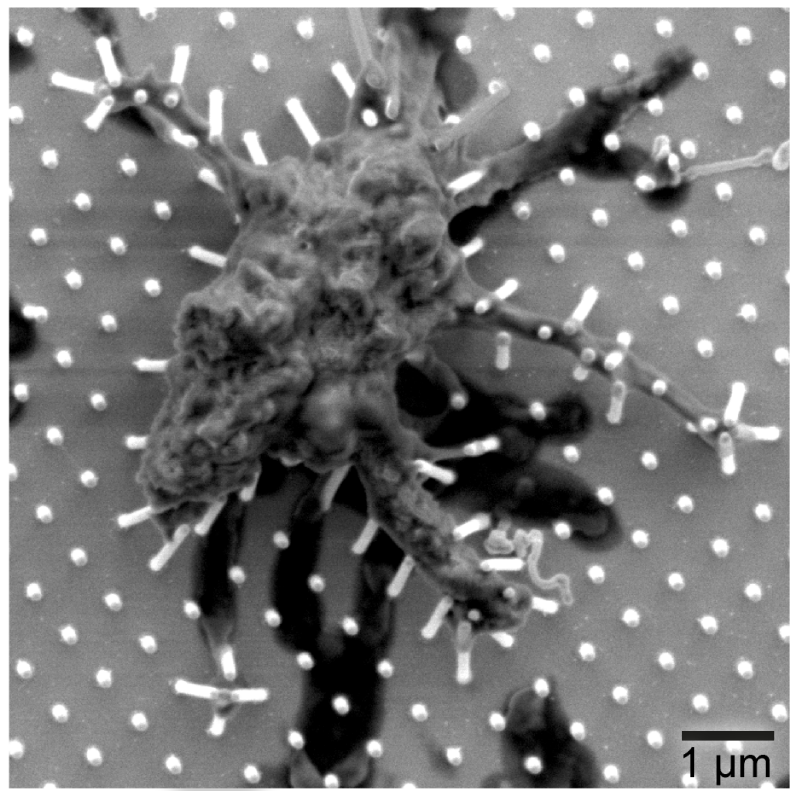

B

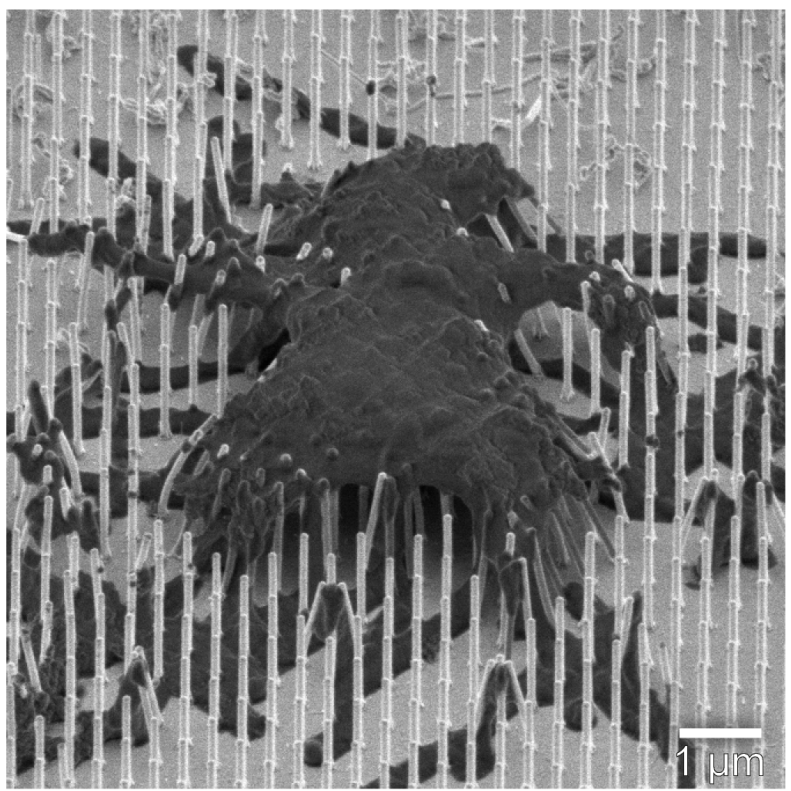

Figure S7. FESEM images of dry, small biofilms on InP nanowire array in perpendicular (A) and tilted (B) view. The EPS matrix covering the biofilm strongly contributes to its integrity and surface adhesion strength. 


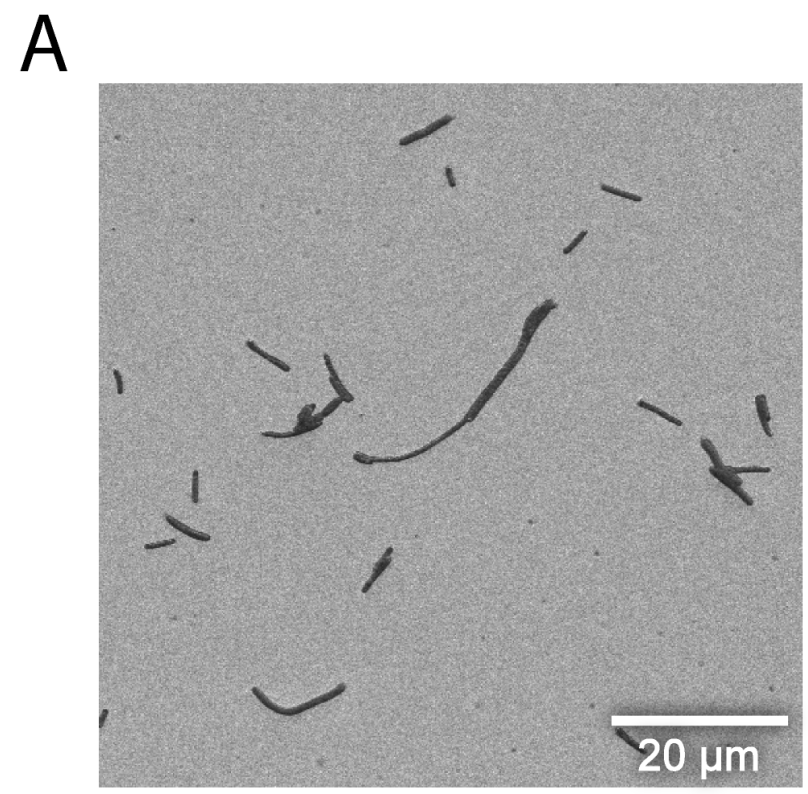

B
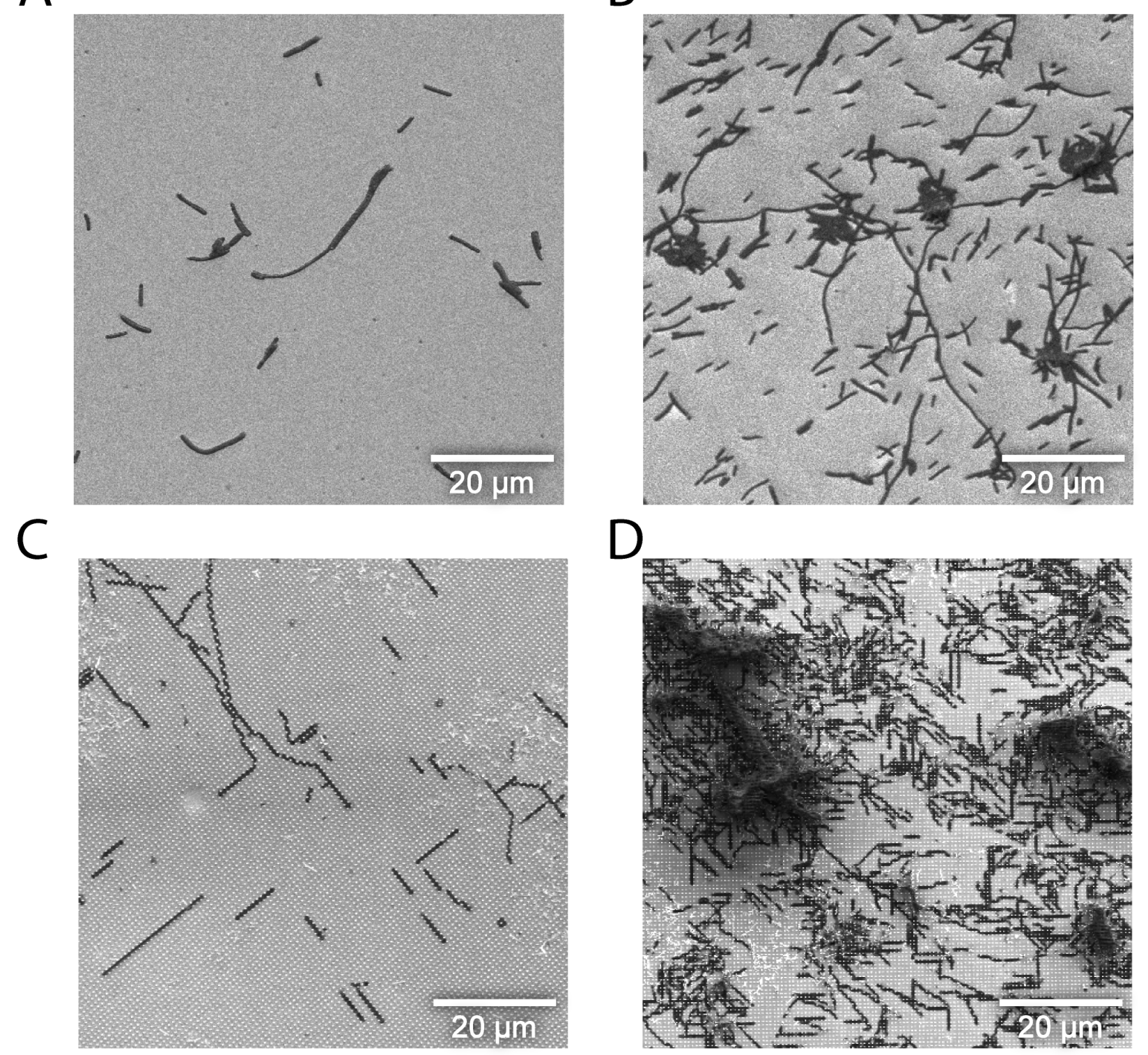

Figure S8. FESEM images of dry $X$. fastidiosa bacterial cells attached to (A) flat InP substrate and (B) flat XadA1-coated InP substrate, with same growth times. Corresponding cell distributions on InP nanowire arrays for non-coated (C) and XadA1-functionalized (D) InP nanowire array. The results indicate that the bacterial cell densities increase significantly upon functionalization with XadA1 and consequently the rapid formation of biofilms. 
A

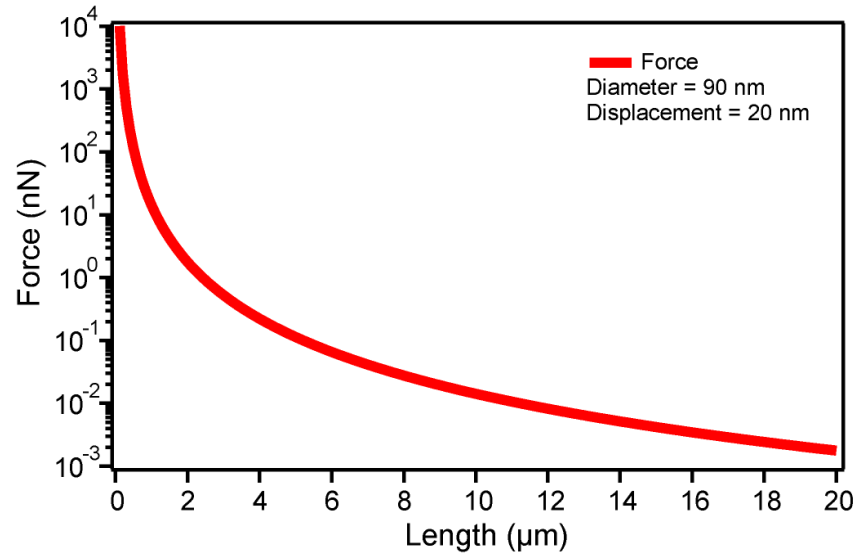

B

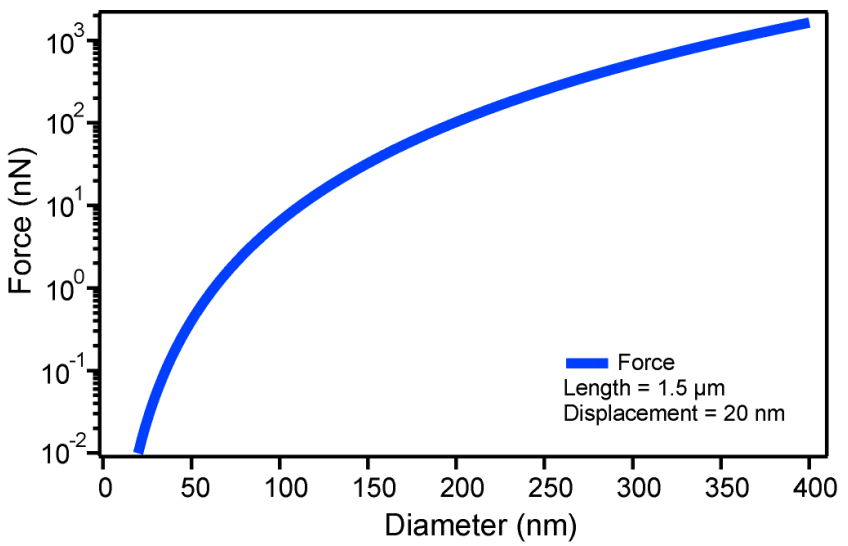

Figure S9. Calculations showing the possibility of measuring the range of bacterial adhesion forces by appropriately choosing (A) length and (B) diameter of InP nanowire arrays. The calculations show that these arrays have the potential to measure a wide range of forces - from $\mathrm{pN}$ to $\mathrm{nN}$ - from bacterial cells. The minimum nanowire tip displacement observable in CLSM measurements was considered for the calculations.

Table S1. Average and maximum adhesion force values (in $\mathrm{nN}$ ) of single cells obtained for all experimental conditions, such as horizontally $(\mathrm{H})$ and vertically $(\mathrm{V})$ moving $(\mathrm{M})$ and static (S) cells on bare and XadA1 $(\mathrm{X})$ coated InP nanowire arrays. For horizontal oriented cells, forces from the bacterial pole and cell body are described with $P$ and $B$, respectively. The force values correspond to the Tukey plot shown in Figure 3G. Obtained forces of control nanowires for every condition are denoted with $\mathrm{C}$.

\begin{tabular}{|c|c|c|c|c|c|c|}
\hline & & Configuration & $N$ & $\begin{array}{c}\text { Average } \pm \\
S D\end{array}$ & Median & $F_{\text {max }}$ \\
\hline \multirow{10}{*}{ 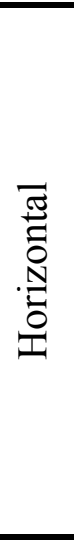 } & \multirow{2}{*}{ Control } & $\mathrm{HC}$ & 222 & $2.6 \pm 1$ & 2.3 & 7.3 \\
\hline & & HCX & 256 & $2.7 \pm 2$ & 2.2 & 7.5 \\
\hline & \multirow{4}{*}{ Static } & $\mathrm{HSP}$ & 137 & $10.2 \pm 8$ & 6.1 & 25.8 \\
\hline & & $\mathrm{HS} B$ & 130 & $7.1 \pm 4$ & 5.7 & 13.6 \\
\hline & & HSXP & 159 & $15.4 \pm 10$ & 12.3 & 42.2 \\
\hline & & HSX B & 144 & $11.5 \pm 7$ & 9.4 & 25.1 \\
\hline & \multirow{4}{*}{ Moving } & $\mathrm{HM} P$ & 65 & $10.1 \pm 5$ & 9 & 20.3 \\
\hline & & $\mathrm{HM} B$ & 137 & $6.9 \pm 5$ & 5.3 & 16.7 \\
\hline & & HMXP & 164 & $15.2 \pm 12$ & 11.1 & 45.4 \\
\hline & & HMXB & 144 & $12.2 \pm 10$ & 8.7 & 31.9 \\
\hline \multirow{6}{*}{ 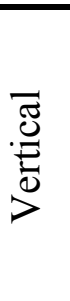 } & \multirow{2}{*}{ Control } & $\mathrm{VC}$ & 123 & $4 \pm 2$ & 3.6 & 8.9 \\
\hline & & VCX & 193 & $5.3 \pm 3$ & 4.9 & 13.8 \\
\hline & \multirow[b]{2}{*}{ Static } & VS & 113 & $9 \pm 5$ & 8.4 & 25.5 \\
\hline & & VSX & 172 & $12.3 \pm 9$ & 9.4 & 43.2 \\
\hline & \multirow{2}{*}{ Moving } & VM & 92 & $8.6 \pm 6$ & 7.3 & 30.2 \\
\hline & & VMX & 141 & $15.8 \pm 10$ & 13.8 & 41 \\
\hline
\end{tabular}


Table S2. Average and maximum adhesion force values (in $\mathrm{nN}$ ) of single cells that anchors the biofilm shown in Figure 2 G.

\begin{tabular}{c|c|c|c}
\hline & Control & Nanowire 1 & Nanowire 2 \\
\hline$N$ & 42 & 42 & 42 \\
\hline Average & 4.6 & 17.1 & 14.2 \\
$S D$ & \pm 3 & \pm 10 & \pm 10 \\
\hline Median & 5.1 & 15.9 & 9.8 \\
\hline $\mathrm{F}_{\max }$ & 10.2 & 34.5 & 32.6 \\
\hline
\end{tabular}

\section{References}

1. Martensson, T.; Carlberg, P.; Borgstrom, M.; Montelius, L.; Seifert, W.; Samuelson L. Nano Lett. 2004, 4, 699-702.

2. Borgstrom, M. T.; Wallentin, J.; Tragardh, J.; Ramvall, P.; Ek, M.; Wallenberg, L. R.; Samuelson, L.; Deppert, K. Nano Res. 2010, 3, 264-270.

3. Gao, L.; Cui, Y.; Wang, J.; Cavalli, A.; Standing, A.; Vu, T. T. T.; Verheijen, M. A.; Haverkort, J. E. M.; Bakkers, E. P. A. M.; Notten, P. H. L. Nano Lett. 2014, 14, 3715-3719.

4. Moreau, A. L. D.; Janissen, R.; Santos, C. A.; Peroni, L. A.; Stach-Machado, D. R.; de Souza, A.A.; de Souza, A. P.; Cotta, M. A. Biosensors \& Bioelectronics 2012, 36, 62-68.

5. Jacobson, B.S.; Fairman, K.R. Anal. Biochem. 1980, 106, 114-117.

6. Hermanson, G.T. Bioconjugate Techniques (Academic Press: Amsterdam, 2013)

7. Davis, M. J.; French, W. J.; Schaad, N. W. Current Microbiology 1981, 6, 309-314.

8. Janissen, R.; Murillo, D. M.; Niza, B.; Sahoo, P. K.; Nobrega, M. M.; Cesar, C. L.; Temperini, M. L.; Carvalho, H. F.; de Souza A. A.; Cotta, M. A. Sci. Rep. 2015, 5, 9856.

9. Caserta, R.; Takita, M. A.; Targon, M. L.; Rosselli-Murai, L. K.; Souza, A. P.; Peroni, L.; StachMachado, D. R.; Andrade, A.; Labate, C. A.; Kitajima, E. W.; Machado, M. A.; de Souza, A. A. Appl. Env. Microbiology 2010, 76, 4250-4259.

10. Schneider, C. A.; Rasband, W. S.; Eliceiri, K. W. Nat. Methods 2012, 9, 671-675.

11. Landau, E. D.; Lifshizt, E. M. Theory of Elasticity (Pergamon Press: New York, 1959).

12. Hallstrom, W.; Lexholm, M.; Suyatin, D. B.; Hammarin, G.; Hessman, D.; Samuelson, L.; Montelius, L.; Kanje M.; Prinz, C. N. Nano Lett. 2010, 10, 782-787.

13. Landau, E. D.; Lifshizt, E. M. Theory of Elasticity (Pergamon Press: New York, 1959).

14. Santos, C. L.; Piquini, Paulo. Phys. Rev. B 2010, 81, 075408.

15. Frigge, M.; Hoaglin, D. C.; Iglewicz, B. The American Statistician 1989, 43, 50-54. 